\title{
Oseltamivir was safe and effective for prophylaxis of influenza in the frail elderly
}

Peters PH Jr, Gravenstein S, Norword P, et al. Long-term use of oseltamivir for the prophylaxis of influenza in a vaccinated frail older population.J Am Geriatr Soc 2001 Aug;49:1025-31.

\section{QUESTION: In frail older people in a residential setting, is oseltamivir (a neuraminidase inhibitor) safe and effective in preventing influenza $A$ and $B$ ?}

Design

Randomised (allocation concealed*), blinded (clinicians and participants),* placebo controlled trial with 8 weeks of follow up.

\section{Setting}

31 residential homes or sheltered accommodations for seniors in 5 countries during the 1998-99 influenza season.

\section{Participants}

572 people who were $\geqslant 65$ years of age, resided in care homes for seniors, and scored $\geqslant 7$ on a Mental Status Questionnaire. Exclusion criteria included antibiotic treatment for acute upper respiratory tract infection, otitis media, bronchitis, or sinusitis, and antiviral treatment for influenza in the previous 2 weeks. 548 people (mean age 81 y, $69 \%$ women), of whom $80 \%$ were vaccinated against influenza, received $\geqslant 1$ dose of study drug and were included in the intention to treat analysis.

\section{Intervention}

Participants were stratified by influenza vaccination status and co-existing chronic obstructive airways disease, and allocated to oseltamivir $75 \mathrm{mg}$ once daily for 6 weeks $(n=276)$ or placebo $(n=272)$ when local influenza activity was detected.

\section{Main outcome measures}

Laboratory-confirmed clinical influenza (temperature $\geqslant 37.2^{\circ} \mathrm{C}$ plus 1 respiratory symptom [cough, sore throat, or nasal congestion] and 1 constitutional symptom [aches and pains, fatigue, headache, or chills or sweats]). Secondary outcomes were symptomatic laboratoryconfirmed influenza not meeting the criteria for clinical influenza, asymptomatic laboratory-confirmed influenza infection, influenza-like illness, and complications of influenza.

\section{Main results}

Fewer patients who received oseltamivir had laboratoryconfirmed clinical influenza than did those who received placebo $(\mathrm{p}=0.002)$ (table). The groups did not differ for laboratory-confirmed influenza, including all clinical cases; influenza not meeting clinical criteria; and asymptomatic influenza $(\mathrm{p}=0.18)$ or acute respiratory illness other than influenza $\{\mathrm{p}=0.75\} \uparrow$. Fewer oseltamivir-group patients had complications of influenza than did placebo-group patients $(\mathrm{p}=0.037)$ (table). The groups did not differ for adverse events (84\% v 89\%), 90\% of which were of mild-to-moderate intensity.

78238, USA.

Oseltamivir v placebo for influenza prophylaxis in frail older people

\begin{tabular}{lllll}
$\begin{array}{l}\text { Outcomes at } 8 \text { weeks } \\
\begin{array}{c}\text { Laboratory-confirmed } \\
\text { clinical influenza }\end{array}\end{array}$ & Oseltamivir & Placebo & RRR (95\% Cl) & NNT (Cl) \\
\hline Influenza complications & $0.4 \%$ & $4.4 \%$ & $92 \%(51$ to 99$)$ & 25 (14 to 56$)$ \\
\hline
\end{tabular}

¥Abbreviations defined in glossary; NNT and $\mathrm{Cl}$ calculated from data in article.

\section{Conclusion}

In frail older people, oseltamivir was safe and effective in preventing influenza $\mathrm{A}$ and $\mathrm{B}$.

*See glossary.

$\dagger \mathrm{p}$ Value calculated from data in article.

\section{COMMENTARY}

Residents of long term care facilities for the elderly are at high risk for influenza-related complications. ${ }^{1}$ In addition, because influenza vaccine is of limited efficacy in frail older people, ${ }^{2}$ nursing home influenza outbreaks remain common despite routine vaccination of residents; therefore, we need to know whether additional benefit exists for antiviral prophylaxis in this setting.

The study by Peters $e t$ al shows that oseltamivir is effective in preventing febrile illness caused by influenza and influenza complications in the elderly. Reducing complications is important because the complications of influenza rather than the disease itself are the cause of death and admission to hospital. In contrast to studies in younger populations, ${ }^{3}$ no excess of nausea and vomiting occurred in the oseltamivir group in this study. Oseltamivir thus joins the list of medications that are effective in preventing influenza and its complications in residents of long term care facilities; the others are the M2 inhibitors, amantadine and rimantadine, as well as zanamivir - another neuraminidase inhibitor.

Both Canadian and US guidelines recommend the use of antiviral prophylaxis during influenza outbreaks. ${ }^{45} \mathrm{Neu}-$ raminidase inhibitors are clearly indicated in situations in which M2 inhibitors are ineffective (outbreaks caused by influenza B and those caused by influenza A resistant to M2 inhibitors) or for patients in whom M2 inhibitors are contraindicated. For routine prophylaxis against influenza A, the appropriate choice of antiviral agent is not as clear. Neuraminidase inhibitors are associated with fewer side effects and less selection for resistance than are M2 inhibitors. However, whether these differences are substantial enough to warrant the additional cost of a neuraminidase inhibitor is unknown.

Allison McGeer, MD, MSc Mount Sinai Hospital Toronto, Ontario, Canada

1 Gross PA, Hermogenes AW, Sacks HS, et al. The efficacy of influenza vaccine in elderly persons. A meta-analysis and review of the literature. Ann Intern Med 1995;123:518-27.

2 Stevenson CG, McArthur MA, Naus M, et al. Prevention of influenza and pneumococcal pneumonia in Canadian longterm care facilities: how are we doing? CMAJ 2001;164: 1413-9.

3 Hayden FG, Gubareva LV, Monto AS, et al. Inhaled zanamivir for the prevention of influenza in families. $N$ Engl J Med 2000;343:1282-9.

4 Centers for Disease Control and Prevention. Prevention and control of influenza: recommendations of the Advisory Committee on Immunization Practices. MMWR Morb Mortal Wkly Rep 2000;49:1-38.

5 National Advisory Committee on Immunization (NACI), Health Canada. Statement on influenza vaccination for the 2000-2001 season. Can Commun Dis Rep 2000;26:1-16. 